\title{
An ontology-based consultation system to support medical care on board seagoing vessels
}

\section{Gabriele Carletti ${ }^{1}$, Paolo Giuliodori ${ }^{2}$, Vincenzo Di Pietri ${ }^{3}$, Alessandro Peretti ${ }^{1}$, Francesco Amenta ${ }^{1,3}$}

\author{
${ }^{1}$ Telemedicine and Telepharmacy Centre, University of Camerino, Camerino, Italy \\ ${ }^{2}$ School of Science and Technology, Computer Science Division, University of Camerino, Camerino, Italy \\ ${ }^{3}$ Research Department, International Radiomedical Centre (CIRM), Rome, Italy
}

\begin{abstract}
Background: A realistic possibility to obtain medical care for patients located in remote sites such as seagoing vessels, in which health professionals are not available, is to contact a doctor via telecommunication systems. In general, the medical knowledge of who on board ships is in charge of medical care is quite limited and therefore, in a first level telemedical consultation, the flow of information should be correct and its efficiency should be maximised. This paper describes an application conceived to improve requests of medical assistance from sailing ships. The ultimate objective of this system is a) to standardise as much as possible the requests of medical advice at a distance, $b$ ) to overcome language barriers and jammed-related troubles that could make difficult or not understandable a telephone conversation. Materials and methods: The application is based on a software engine extracting data from an ontological knowledgebase built ad hoc using Protégé.

Results: Compared to the conventional consultation systems based on telephone and e-mail, the proposed device is more accurate and complete in terms of information contained in the request of assistance. Moreover, data received by the medical centre can be more easily managed, as they can be standardised. Conclusions: The system described here allows people responsible of medical care on board ships to forward detailed requests of assistance containing symptom-guided information on patient clinical conditions. This may represent an innovative tool for medical consultations at distance allowing the remote centre to provide more precise and quicker medical advice.
\end{abstract}

(Int Marit Health 2016; 67, 1: 14-20)

Key words: teleconsultation, remote medical advice, ontology, Protégé, medical assistance on board ships

\section{INTRODUCTION}

Telemedicine, consisting in the application of information and communication technology (ICT) to the solution of medical problems and in the exchange of medical information, is changing our approach in the delivery of several health services. Telemedicine includes a growing variety of applications and services the use of which will increase in the near future.

Teleconsultation (e.g. the medical visit made via ICT) can be divided into different levels based on the players involved and the complexity of the information exchanged. A basic (first level) teleconsultation is the electronic/telephonic communication between a client (patient) and a physician. A second level teleconsultation involves a physician or another health professional and a specialist delivering health care services and information over small and large distances. In the second level teleconsultation, data, information, images and/or voice are exchanged. A higher level of teleconsultation (third level) involves a medical team of a hospital addressing specific questions to the team of

Dr Francesco Amenta, Centro di Telemedicina e Telefarmacia, Via del Bastione, 62032, Camerino, Italy, e-mail: francesco.amenta@unicam.it

Paper presented at the Meeting "The Way Forward of Maritime Telemedicine" held in Rome on October 30-31, 2015 as an activity in the occasion of celebrations for the $80^{\text {th }}$ Anniversary of Centro Internazionale Radio Medico (CIRM) 
a specialised centre. In primary care, generally, teleconsultation takes place between a patient/caregiver and health care professionals for diagnostic or therapeutic advice or for educational purposes.

Medical assistance of good quality is a right of all citizens, but it is not always easily deliverable in remote areas, such as seagoing vessels as well as small islands, rural regions, or in developing countries. Remote teleconsultation therefore represents the only realistic way to deliver health care to patients not able to obtain direct medical assistance such as seafarers on board ships without medical facilities. Seagoing vessels represent a prototype of isolated place, and could remain at sea for days or weeks before reaching a port. The largest majority of merchant ships do not carry doctors or expert paramedic personnel and an officer with medical duties (the captain or the first mate) is the person in charge of the patient in case of accidents or diseases. For more than 80 years, several radio medical services have been operational, starting by using radio signals and Morse code [1], evolving through telephones to full blown telemedicine solutions. Today specialised ashore centres called Telemedical Maritime Assistance Service (TMAS) offer medical assistance to ships with no doctor on board [2]. Telemedical consultations, however, have some innate limitations. One consists in the fact that the great majority of people asking for medical advice by means of ICT resources do not have proper medical training. The communication of symptoms or specific clinical situations can therefore be difficult or misleading in case of absence of objective information such as biomedical data and/or video support [3].

This paper presents a system conceived to improve the first level teleconsultation by allowing the correct flow of the relevant information on the status of a patient, avoiding problems related to verbal communication or jammed transmission. This system guides the ship captain in the medical examination, increasing the preciseness of the information transmitted with the consequent possibility to obtain more accurate and quicker diagnosis.

Potential users of the system besides seafarers could be also other isolated populations such as personnel on board of commercial aircrafts or oil-rig workers or people living in rural areas. Hence, a potential high number of users can benefit from the system that will allow, starting from a given sign, the identification of the cohort of other signs and symptoms present.

\section{MATERIALS AND METHODS}

\section{THE KNOWLEDGE BASE}

A knowledgebase was built in the shape of an ontology. The ontology represents concepts and their logical relations and has a hierarchical structure. It is a suitable solution for data managing and sharing, allowing a universal codification of concepts. In this sense, the effort of the Open Biological Ontologies (OBO) Foundry to create a set of interoperable ontologies enabling scientists and their instruments to communicate with minimum ambiguity should be mentioned [4]. An ontology also permits the reuse of knowledge and the inference of new knowledge, through automatic reasoning $[5,6]$.

In the present work this ontology has been developed using the existing software Protégé 3.4.1 [7], aggregating free-text data from the relevant literature [8]. When possible, our terms for clinical signs were associated as synonyms with the ones from SYMP (the OBO Foundry Symptom Ontology) [6] exploiting the "Bioportal" function of Protégé that allows linking to external resources.

Two main classes were created: "Sign" and "Detailed info". A series of instances for the class "Sign" has been created to represent the main signs a patient could manifest, in example "Fever", "Cough", "Breathing difficulties", "Diarrhea”, etc. Each main sign has relations (object property "hasDetailedInfo" in the ontology) with a series $(1, n)$ of detailed information to be communicated to the medical centre. These information are represented in the ontology as instances of the class "Detailed info", or better, of its subclasses.

Each main sign has relations (object property "hasAdditionalSign" in the ontology) with a series $(1, n)$ of other signs (other instances of the class "Sign"). For instance, "Fever" has relations with "Cough", "Breathing difficulties", "Diarrhea", etc., that represent possible associated clinical signs requested in the final application to the patient to be considered selecting "Fever" as main sign (Figs. 1, 2).

In the same way, to each detailed information has been assigned an answer that finally would represent the option the user should select or fill in the final application. A SPARQL query has been then created to retrieve data from the ontology. The following represents the general syntax to retrieve the detailed information and their relevant superclasses for the main sign "Fever" i.e.:

"SELECT ?detail ?answer ?superclass

WHERE $\{$ :Fever :hasDetailedInfo ?detail.

?detail rdf:type ?superclass \}".

\section{THE APPLICATION}

A software application was then built as the engine to interact with the “.owl” generated Protégé file.

\section{Basic software technology and JSP}

The system is based on a web service implemented in JSP. JSP seemed to be the most suitable solution, providing a Java library capable of interacting with the OWL standard. Java methods and classes can easily be called in a JSP page. The following are the main JSP pages (Fig. 3). 


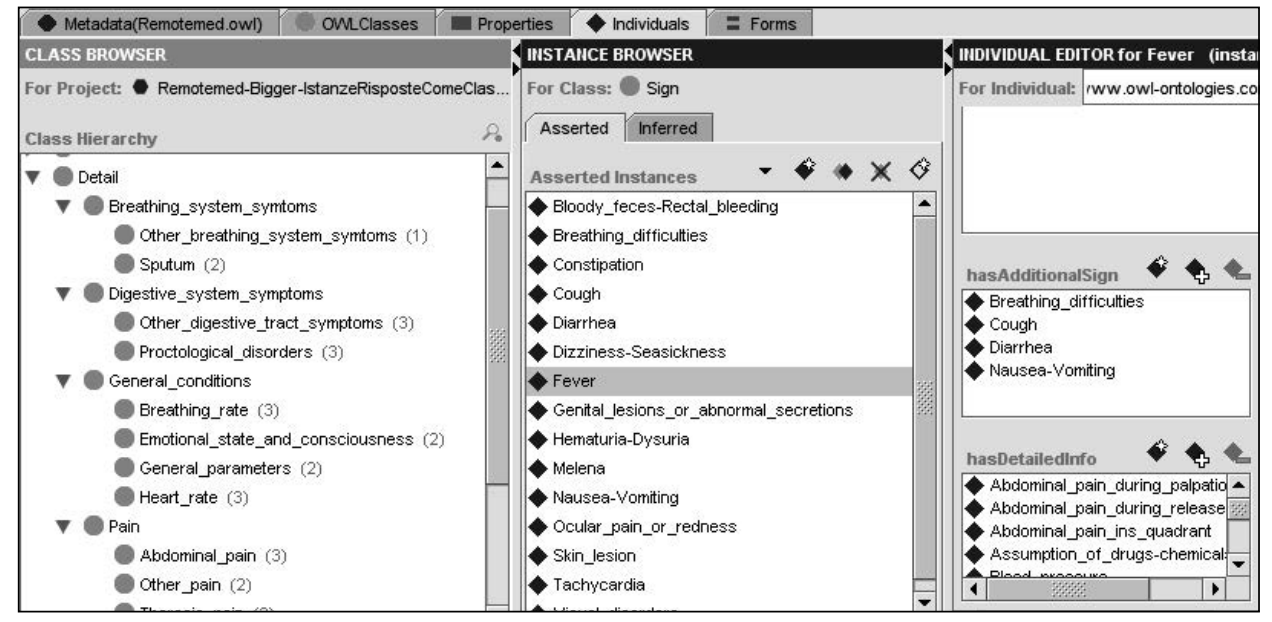

Figure 1. Protègè - some of the detailed information and additional signs (in the right) for the instance "Fever". Some of the superclasses in the left

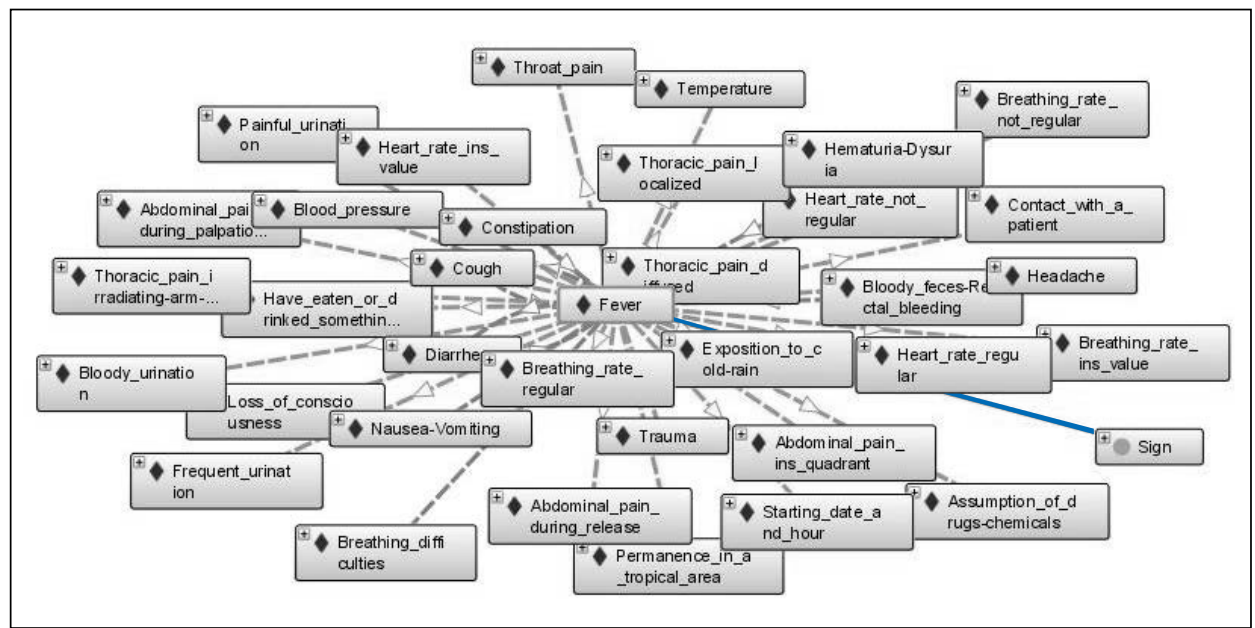

Figure 2. OntoGraph showing Additional Signs (blue line) and Detailed Info for the sign "Fever"

The system allows for saving data in an XML database:

- main.jsp, main page that allows the user to select the principal sign and answer the detailed information and the additional signs; it contains all the JavaScript functions related to the web service, such as the "Yes/No" functionality (Fig. 4);

- save.jsp, process page that saves the information inserted by the user in the XML database;

- menu.jsp, a navigation page;

- style.css, defines the CSS style of the elements.

The system even allows the doctors of the medical centre to answer the requests inserting a diagnosis and the admin to edit saved data. These are the relevant JSP pages:

- save_diagnosis.jsp, process page that saves the diagnosis inserted by the doctors in the XML database;

- view_data.jsp, allows doctors and admin to see and edit all the records. Admin could then, for instance, decide to delete one or more users' records and doctors to add or modify a diagnosis;

- delete_users.jsp, process page that permits to delete users' records from the XML file.

The two principal pages concerning the final user (patient, doctor or admin) are "main.jsp" and "view_data.jsp", the first to insert, the second to view data. The other pages, excluding "menu.jsp", are processing pages and have the task to interact with the XML database and to modify it.

\section{The Java classes}

The Java classes work as interface between the application, the ontology and the XML database (Fig. 3). The main Java classes used were:

- OWLAPI, it acquires all the information contained in the ontology. For instance, when the page "main.jsp" is loaded, this Java class gets all the principal signs or when a user selects a principal sign, a method of the OWLAPI acquires 


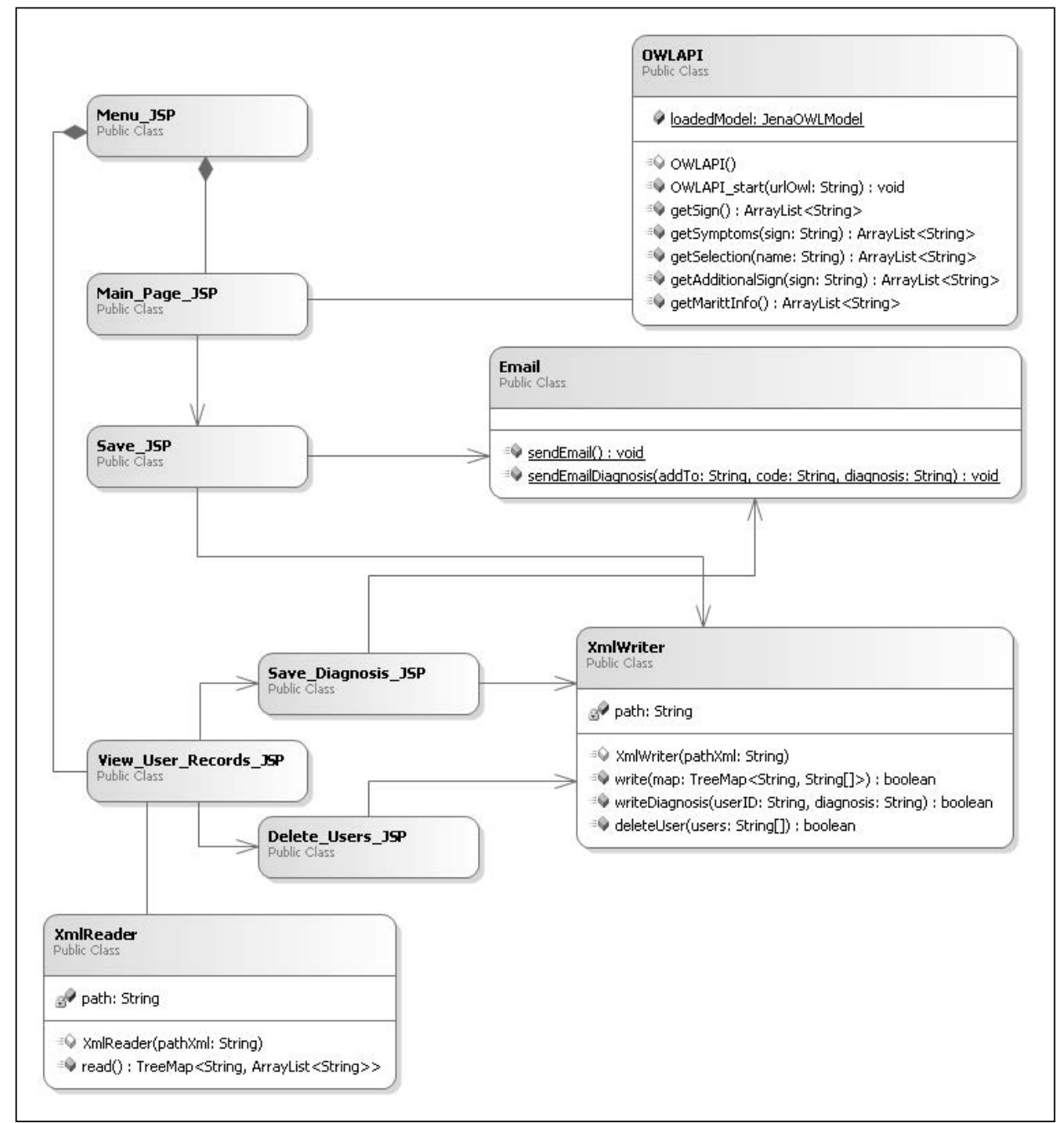

Figure 3. The main JSP pages and Java classes

\begin{tabular}{|c|c|}
\hline (Fill out all fields and then click on submit.) & \\
\hline Principal sign: Fever & $\checkmark$ \\
\hline Abdominal pain $Y e s /$ No & \\
\hline Breathing rate $Y e s / N o$ & \\
\hline Emotional state and consciousness Yes/No & \\
\hline General parameters $Y$ Yes/No & \\
\hline Heart rate Yes/No & \\
\hline $\begin{array}{lll}\text { NamedIndividual } & \text { Yes/No } \\
\end{array}$ & \\
\hline Other pain $Y$ Yes $/ N_{0}$ & \\
\hline Presuntive causes Yes/No & \\
\hline Starting information $Y$ Yes/No & \\
\hline Thoracic pain $Y e s / N_{0}$ & \\
\hline Urinary functions $\mathrm{Yes}_{\mathrm{N}} \mathrm{No}$ & \\
\hline Additional Sign: & \\
\hline Breathing difficulties & $\square$ Yes \\
\hline Cough & $\square$ Yes \\
\hline Diarrhea & $\square$ Yes \\
\hline
\end{tabular}

Figure 4. Groups of detailed information (ontological superclasses) to be explored/opened for the main sign "Fever" from the ontology all the related detailed information and additional signs. This class takes advantage of the Protégé Java Library that permits to interact with an RDF/XML model that in fact is the structure of the ontology;

- Email, the task of this class is to send email to the medical centre with the list of information inserted by the user and to the user if a doctor has inserted or modified a diagnosis;

- XmIReader, this class is called when the page "view data.jsp" is loaded. It takes all the records contained in the XML database;

- XmIWriter, as XmIReader it interacts with the XML database and inserts users' records and doctors' diagnosis.

\section{THE PATIENT'S USER INTERFACE \\ Detailed info navigation and answering}

The hierarchical organisation of concepts and the superclasses make easier and less time-consuming for the user to navigate the information. Selecting the main sign, the system asks to consider a series of additional signs and detailed infor- 


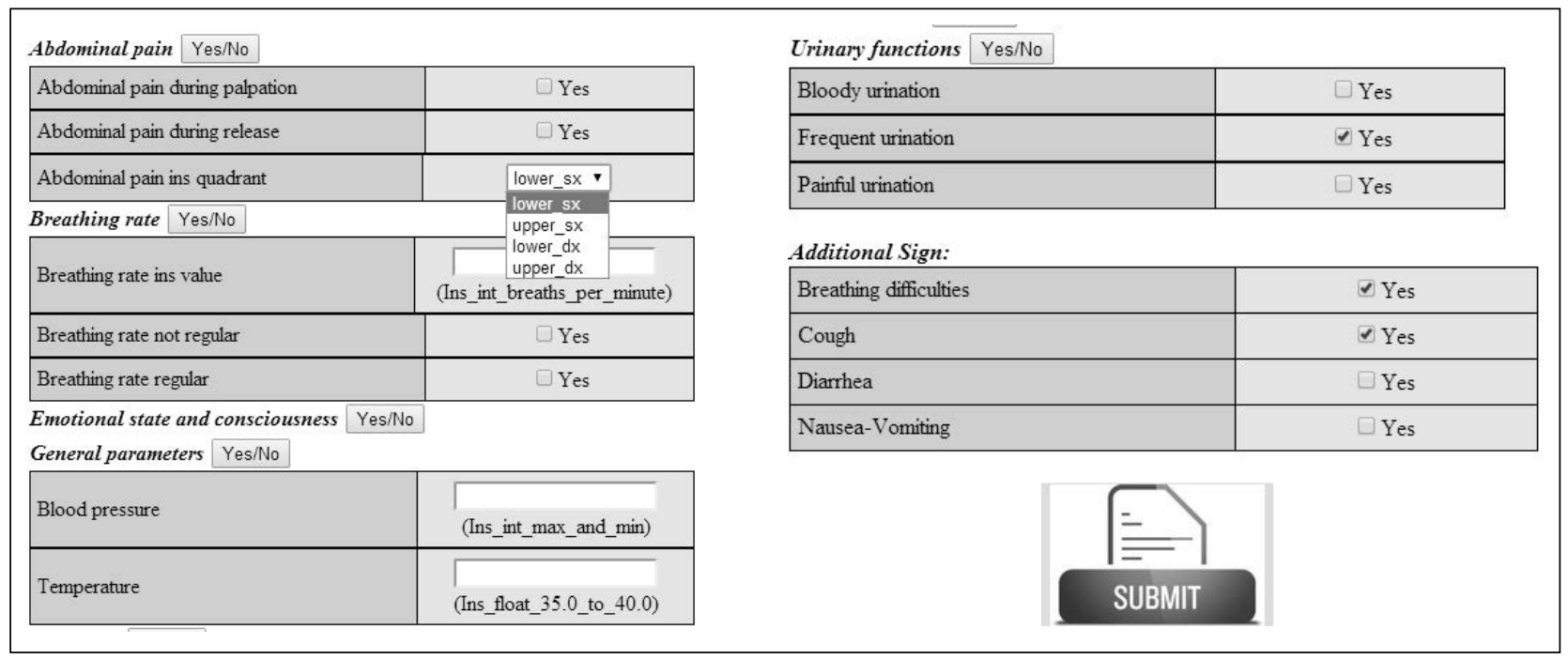

Figure 5. Left part shows a detailed information referring to each superclass with the relevant answer options to select or fill in. The right part of the figure shows the procedure ends with the submission of the compiled form

mation. These are organised in big groups (superclasses). If the patient doesn't manifest the sign to which the group is referring or doesn't want to consider that superclass, he can simply answer "No" (or don't click) to the relevant superclass (Fig. 4).

By clicking on a superclass, the series of detailed info about that clinical status appears. They can be answered in the following three main ways (Fig. 5):

- boolean selection (Yes/No);

- multiple selection;

- typing (generally a string or a number).

\section{Other information}

Patient's general info. Some basic details on the patient needing medical assistance and simple information on his personal history should be provided:

- age;

- name;

- birthdate;

- sex;

- nationality;

- rank.

Patient's clinical history and drugs administered. Basic medical history and drug history of the patient should be included in the following order:

- previous most significant diseases;

- chronically administered drugs;

- drugs administered for the actual condition.

\section{Submission of data}

Once answered all the detailed information referring to all the superclasses as described above, the user can submit them, sending the data package to the maritime telemedical centre (TMAS) (Fig. 5).
The system has been adapted in particular for naval communication. Then, a series of information about the ship is required to be inserted, such as:

- ship name;

- ship type;

- ship owner;

- call sign;

- master;

- ship nationality;

- port of departure;

- port of arrival;

- speed;

- position;

- telephone/fax/e-mail.

\section{SYSTEM TESTING}

Tests of the system are ongoing in collaboration with Centro Internazionale Radio Medico (CIRM), the Italian TMAS [9] using seagoing vessels as a prototype of isolated places. CIRM medical assistance is given to ships of any nationality sailing worldwide. The service is provided $24 \mathrm{~h}$ a day and 365/366 days per year by doctors on duty. The centre receives the request of assistance and gives instructions for the case. For assessing the quality of the system, 150 teleconsultations between seagoing participating voluntarily to the experiments (users) and CIRM medical team (experts), were evaluated. Evaluation of the system involved both the user's and the expert's side and both user's satisfaction and technical aspects. The parameters listed below were considered compared to standard communication systems:

- accuracy of the request (number of non-ambiguous signs communicated per request); 
Table 1. Evaluation of the system effectiveness using 150 teleconsultations from seagoing vessels (user) and Centro Internazionale Radio Medico (CIRM) headquarters in Rome (expert)

\begin{tabular}{lll}
\hline Parameter & Tester & Score \\
\hline Accuracy of the request & Expert & High \\
Accuracy of the possible diagnosis & Expert & High \\
Speed - overall & User-Expert & Medium \\
Speed - diagnosis & Expert & High \\
Usability & User & High \\
Wiliness to use & User & Medium \\
Completeness of the info & User-Expert & High \\
Easiness of data managing & Expert & High \\
Reliability of the system & Expert & High
\end{tabular}

"High", "Medium" and "Low" indicate respectively for better, same as, and worse compared to standard (telephone or e-mail) previous communication systems.

- accuracy of the diagnosis made by the centre (number of correct verified diagnosis/total cases);

- speed of a complete round: time to make the request/ /time to provide the answer;

- speed of the diagnosis;

- usability (easiness of use, even by an inexpert user);

- wiliness to use;

- completeness of the information;

- overall reliability of the system.

A score from "High" to "Medium" to "Low" was assigned by the testers (users and experts) for each of the above parameters.

\section{RESULTS}

The system described here has shown the capability to forward accurate remote requests of assistance with no technical problems in terms of software functionality and integrity of data transmission.

Differently to other tools that are diagnosis-oriented [10], the proposed system does not provide diagnostic solutions, but results efficient in guiding the user towards the collection of appropriate signs, already codified according to the guidelines provided by the maritime telemedical centre, with no possibility for self-ambiguity and ensuring the information transmitted to be as complete as required.

Table 1 summarises the results reporting the prevalent mark on the total number of evaluations provided by the testers. The system has shown, in respect to the existing consultation tools, a higher completeness and accuracy of the request of assistance in terms of information transmitted, a quicker and more accurate diagnostic possibility and an overall high usability and reliability.

\section{DISCUSSION AND CONCLUSIONS}

The system allows the user to prepare telemedical requests of advice with precise and circumstantial informa- tion. A substantial advantage derived from adopting the application for telemedical consultation consists in the fact that a great number of specific information about the patient (automatically suggested starting from the main symptom) could be transmitted to the physicians in charge of medical assistance, increasing the chance to get a correct and faster advice. Moreover, data transmitted are encoded using a standard vocabulary and a standard formulation. The information transmitted is therefore more easily managed by the centre. The system will also contribute to constitute a patient's historical data repository as a support for further teleconsultations.

Thanks to the ontologies, an easy to obtain multi-language selection is available. This option could reduce communication barriers caused by language troubles, misunderstandings and verbal hesitations mostly due to the unavoidable use of technical clinical terms. Problems of jammed telephonic communication are also eliminated through the direct transmission of data.

As a result, the user is able to get a faster and more accurate answer of assistance from the maritime telemedical centre. Referring to the maritime assistance, a correct and quick diagnosis could avoid unnecessary transfers of the patients, limiting costs for changes of course/evacuations and discomforts for the crew.

In 2013, CIRM reported several problems in providing correct diagnosis due to imprecise information from the ship side. The test of the support communication system described here, showed an improved definition of the medical problems to be treated. The potential advantages of the system appear therefore promising.

\section{FUTURE IMPLEMENTATIONS}

As a possible future enhancement of the system, we are considering the possibility of crowdsourcing the requests, through a server, from the mobile terminal of the patient 


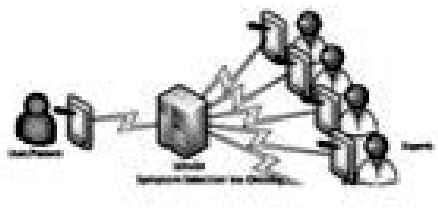

Initial learning phase

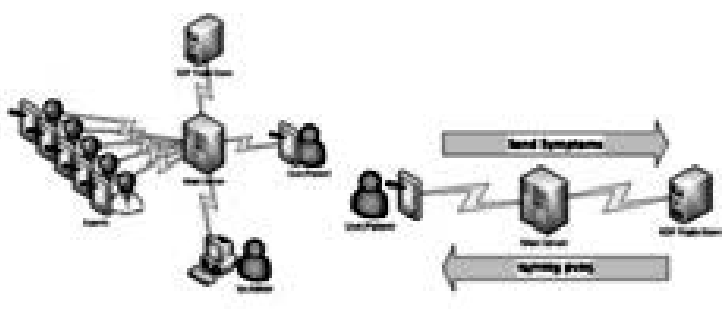

Later automatic phase

Figure 6. General architecture showing the flow of the information for a future possible distribution system. The users' request dispatched to the server would be redistributed to a set of experts and the answers would be both returned to the user and stored into a knowledgebase creating the basis for automatic diagnosis

(a mobile version should be first developed) to a pool of clinicians. Once the clinicians will answer the requests, these data will be returned back to the patients and stored into a knowledgebase (as actually for the XML database), creating the fundamentals for further automatic processing of medical information (Fig. 6).

This will allow the development of a medically-validated, punctiform, status-diagnosis association. In the second automated phase, the clinicians' work will be significantly reduced, but reliable medical answers could be still obtained since they will be inferred from researches and learning processes on the knowledgebase.

Considering the application, it would be interesting to investigate the possibility to perform automatic reasoning on the main ontology in order to infer new knowledge and to rearrange the existing data structure such as the subclasses hierarchy and the positioning of the instances.

\section{ACKNOWLEDGEMENTS}

This research was supported in part by a grant of Italian Ministry of Agricultural, Food and Forestry Policies (contributions in support of the fishing sector).

\section{REFERENCES}

1. Goethe WHG, Watson EN, Jones DT eds. Medical care on ships without a doctor. Radio medical advice. Handbook of Nautical Medicine, Springer, Berlin 1984; 53-65.

2. Medical Assistance at Sea. International Maritime Organization (IMO). Circular MSC/Circ.960. IMO, London 2000.

3. Sabesan S, Allen D, Caldwell P et al. Practical aspects of telehealth: doctor-patient relationship and communication. Intern Med J 2014; 44: 101-103.

4. The OBO Foundry. www.obofoundry.org. (accessed 5 Feb 2014).

5. Köhler S, Bauer S, Mungall CJ et al. Improving ontologies by automatic reasoning and evaluation of logical definitions. BMC Bioinformatics 2011; 12: 418.

6. Nageba E, Fayn J, Rubel P. A knowledge model driven solution for web-based telemedicine applications. Stud Health Technol Inform 2009; 150: 443-447.

7. Protégé documentation. http://protege.standford.edu. (accessed 5 Feb 2014).

8. Camerucci S. I call the CIRM. A manual of first aid and medical assistance for sailors, 2005.

9. Amenta F. The International Radio Medical Centre (C.I.R.M.): an organization providing free medical assistance to seafarers of any nationality world wide. Int Marit Health 2000; 51: 85-91.

10. Luger TM, Houston TK, Suls J. Older adult experience of online diagnosis: results from a scenario-based think-aloud protocol. J Med Internet Res 2014; $16: 1$. 